\title{
In vitro fertilisation with frozen-thawed bovine sperm sexed by flow cytometry and validated for accuracy by real-time PCR
}

\author{
Roberto Puglisi, Roberta Vanni, Andrea Galli, Donatella Balduzzi, Katia Parati, \\ Graziella Bongioni, Gabriella Crotti ${ }^{1}$, Roberto Duchi ${ }^{1}$, Cesare Galli ${ }^{1,2}$, Giovanna Lazzari ${ }^{1}$ and \\ Riccardo Aleandri
}

Istituto Sperimentale Italiano Lazzaro Spallanzani, Viale Forlanini 23, 20134 Milan, Italy, ${ }^{1}$ Laboratorio di Tecnologie della Riproduzione, Istituto Sperimentale Italiano Lazzaro Spallanzani, CIZ srl, via Porcellasco 7/f, 26100 Cremona, Italy and ${ }^{2}$ Dipartimento Clinico Veterinario, Università di Bologna, Via Tolara di sopra 50, 40064 Ozzano Emilia (Bologna), Italy

Correspondence should be addressed to R Puglisi who is now at Istituto Sperimentale Italiano Lazzaro Spallanzani, Loc. La Quercia, 26027 Rivolta d'Adda (Cremona), Italy; Email: isils@tin.it

\begin{abstract}
The methodologies used for cytometric sorting of fresh spermatozoa never allowed a clear resolution of sexual chromosomes of frozen-thawed semen. To devise a novel method for the production of bovine predefined sexed embryos using frozen-thawed semen, sorting efficiency of different protocols was studied using a new quantitative real-time PCR method to verify the purity of sexed semen. To this aim, after Percoll separation, frozen-thawed samples were stained at different temperatures and concentrations of Hoechst 33342 using a short-incubation time. The concentration of Hoechst $33342 \mathrm{of} 500 \mu \mathrm{g} / \mathrm{ml} \mathrm{at} \mathrm{a} \mathrm{temperature}$ of $37{ }^{\circ} \mathrm{C}$ provided good and stable fluorescence signals. Preventing the sperm clustering by adding $0.6 \%$ BSA in the $90 \%$ Percoll fraction led to X-bearing sperms purity of $91 \pm 2 \%$. Thereafter, sorted sperms were used for in vitro fertilisation (IVF). Despite the lower cleavage rates reported in the sorted groups when compared with the control groups (40 vs $48 \%, P<0.01)$, blastocyst formation in the sorted and control groups was not different ( $27 \mathrm{vs} 24 \%$ of the cleaved respectively). The PCR analysis of 30 blastocysts confirmed 26 embryos to be correctly sexed (87\%). Transfer of two embryos per recipient into six synchronised heifers resulted in four pregnancies. Two abortions occurred at day 60, while two pregnancies went to term delivering two female calves. In conclusion, high purity and repeatability of sorting was obtained with frozen-thawed bull semen that was subsequently used for IVF giving rise to viable embryos and offspring. In addition, real-time PCR revealed to be an optimal support for these studies, providing a rapid and reliable estimation of flowcytometric efficiency.

Reproduction (2006) 132 519-526
\end{abstract}

\section{Introduction}

Shifting the sex ratio of a sperm population into $X$ - or Y-bearing sperm samples represents an attainable and powerful mean for the production of predefined sexed embryos and offspring. Thanks to the assisted reproduction technologies, in vitro embryo production is now widely used for rapidly spreading the genetic improvements all over the World and in this optic, the gender preselection would provide an important opportunity to satisfy the needs of the demanding markets and to reduce the genetic defects connected to sex.

Flow cytometric sorting of fresh spermatozoa and their cryopreservation have been used to produce viable offspring in different species (Seidel et al. 1999, Johnson 2000, Hollinshead et al. 2004a). Modifications of the conventional artificial insemination (AI) technology, such as deep-uterine or utero-tubal junction insemination or for low dose $\mathrm{Al}$, have been introduced to compensate the loss of sperm performance derived from the artificial manipulations normally required for sperm sorting (Lindsey et al. 2002, Bodmer et al. 2005, Grossfeld et al. 2005). Nevertheless, the need of having the sperm donor nearby the cytometry laboratory and the difficulties to preserve viability up to the place of insemination, when cryopreservation is not desired, are still the limiting factors for a wide application of this technology.

On the other hand, commercially available frozen-thawed semen exhibits minor tolerance to the stress, reducing considerably the possibilities to apply the sorting procedures to cryopreserved semen 
for in vitro embryo production plans. Some of the critical points still under investigation in the use of frozen semen, are the different methods for cryoprotectant removal, which include washing or density gradients separation, the sperm staining procedures and the pressures and speeds, which can be tolerated by the sperms. Overall, these major concerns increase the effort required, while operating with frozen semen.

A poor response to staining procedures, which results in a minor resolution of $X$ - and Y-bearing sperms, has been reported for frozen-thawed semen with respect to fresh semen (Stap et al. 1998). An increase in both he concentration and the time of incubation with Hoechst 33342 (H33342) has been demonstrated to be beneficial for sorting, although maintenance of the sperms at $34-37^{\circ} \mathrm{C}$ for extended period of time would not be recommended due to the considerable reduction in the proportion of motile and viable spermatozoa (Hollinshead et al. 2004b).

In order to increase the practical use of the technology of sperm sorting and to encourage its application in the field for the production of sexed embryos, the development of a sensible and accurate method to verify the yield in sperm separation is essential. At present, some of the methods described and proven to be applicable in this respect, are represented by flow cytometric reanalysis of the sexed semen (Welch \& Johnson 1999), the analysis by capillary electrophoresis (Checa et al. 2002) and multicolour fluorescence in situ hybridisation (Habermann et al. 2005). However, these methods that were developed along with sperm sorting technologies of fresh semen, are of limited value when frozen-thawed semen is used, considering the series of problems mentioned earlier, which normally result in a minor sorting efficiency and a reduced number of viable frozen-thawed sexed sperms available for use in IVF.

This study is focused on the development of an efficient protocol for in vitro production of predefined sexed embryos using frozen-thawed bull-sorted semen, supported by a new reliable quantitative real-time PCR method to verify the purity of sexed semen.

\section{Materials and Methods}

\section{Sperm preparation}

Frozen semen samples of different bulls were thawed at room temperature for $30 \mathrm{~s}$ in water bath and then selected by 40 min centrifugation at $800 \mathrm{~g}$ on discontinuous Percoll gradients (45-90\%). The 90\% Percoll fraction was prepared by mixing $9 \mathrm{ml}$ Percoll (no. P1644, Sigma-Aldrich) with $1 \mathrm{ml} 10 \times$ Tyrode's solution (46.75 g NaCl/l, $2.3 \mathrm{~g} \mathrm{KCl} / \mathrm{l}, 0.4 \mathrm{~g} \mathrm{NaH}_{2} \mathrm{PO}_{4} / \mathrm{l}, 20.9 \mathrm{~g}$ HEPES/I (pH 7.4)). In the isotonic, 90\% Percoll was then added $2.1 \mathrm{~g} \mathrm{NaHCO} / \mathrm{ll}, 3.7 \mathrm{ml} 60 \%$ Na-lactate/l and $7.8 \mathrm{mg} \mathrm{MgCl}{ }_{2} \cdot 6 \mathrm{H}_{2} \mathrm{O} / \mathrm{l}$. For Percoll gradients preparation,
$2 \mathrm{ml}$ Percoll $90 \%$ were carefully pipetted under $2 \mathrm{ml}$ Percoll $45 \%$ that was obtained by mixing $1 \mathrm{ml}$ Percoll $90 \%$ with $1 \mathrm{ml}$ HEPES-buffered $\mathrm{Ca}^{2+}$ free TALP medium (H-TALP; pH 7.4) supplemented with 0.6\% BSA (no. A9418, fraction V, Lot $129 \mathrm{H} 14205$, Sigma). After centrifugation, the sperm pellets were resuspended in $5 \mathrm{ml} \mathrm{H}$-TALP medium and washed by 10 min centrifugation at $500 \mathrm{~g}$. The pellets were finally resuspended in $500 \mu \mathrm{l}$ of the same H-TALP medium at a concentration of 40 millions of spermatozoa $/ \mathrm{ml}$. During all the procedures, spermatozoa were constantly maintained at room temperature.

\section{Evaluation of different sperm presorting stain treatments}

Different trials were conducted to test the effect of the staining procedure on the ability to resolve $X$ - and Y-chromosomes. To this aim, immediately after Percoll separation, the selected semen samples were stained at different concentrations of the vital nuclear fluorochrome H33342 using a very short period of incubation (15 min in all the trials). The staining procedures were conducted at two different temperatures (room temperature and $37^{\circ} \mathrm{C}$ ). During the first two trials, a concentration of $100 \mu \mathrm{g} / \mathrm{ml} \mathrm{H} 33342$ was tested and the incubations were conducted at room temperature or $37^{\circ} \mathrm{C}$ (experiments 1 and 2 respectively). In the second two trials, the concentration was increased to $500 \mu \mathrm{g} / \mathrm{ml}$ and the incubations were also conducted at both temperatures (experiments 3 and 4). After the staining treatments, the semen samples were immediately flow cytometrically sorted and analysed by real-time PCR to determine the percentage of $\mathrm{X}$ - and $\mathrm{Y}$-chromosome enrichment.

After the analysis of results of these trials, the concentration of $500 \mu \mathrm{g} / \mathrm{ml} \mathrm{H} 33342$ and the incubation at $37^{\circ} \mathrm{C}$ were chosen for a following trial (experiment 5), in which frozen-thawed semen of two different bulls were used and the Percoll gradients procedure was modified by the use of a $10 \times$ Tyrode's solution in which $60 \mathrm{~g}$ BSA/l was dissolved. After dilution, the final concentration of BSA in the $90 \%$ Percoll fraction was $0.6 \%$.

\section{Flow cytometric sorting}

After the incubation with $\mathrm{H} 33342$, the semen was diluted 1:2 (v/v) with H-TALP medium at room temperature and immediately used for cytofluorimetric sorting. A cell sorter (FACS Vantage SE-Becton Dickinson) operating at 20 psi was used. Sperms were excited by an argon laser working at $150 \mathrm{~mW}$ and approximately 1500-2000 sperms/s were selected into X- and Y-bearing sperm samples. The H-TALP medium ( $\mathrm{pH} 7.4)$ not containing BSA was used as sheath fluid, while 
collection vessels coated overnight with H-TALP medium containing 5\% BSA were used for recovering the sorted sperms.

\section{Oocytes recovery and maturation}

Ovaries were collected from a local abattoir and transported to the laboratory within $2 \mathrm{~h}$ in PBS supplemented with penicillin and streptomycin, at $20-25{ }^{\circ} \mathrm{C}$. Follicles of $2-8 \mathrm{~mm}$ diameter were aspirated with a 18 gauge needle connected to a vacuum pump and the oocytes were selected on the basis of morphology and by the presence of homogeneous layers of cumulus cells, in HEPES-buffered TCM 199 medium supplemented with $0.1 \%$ BSA and polyvinyl alcohol (PVA, 0.1\%). Groups of 100-120 selected oocytes were then matured up to $24 \mathrm{~h}$ in $2 \mathrm{ml}$ bicarbonate-buffered TCM 199 supplemented with follicle-stimulating hormone/luteinising hormone $(0.05 \mathrm{UI} / \mathrm{ml}$, Pergovet, Serono) and 10\% FBS (no. F6178, Lot 074K0361, Sigma), in $5 \% \mathrm{CO}_{2}$ and $95 \%$ humidified air at $38.5^{\circ} \mathrm{C}$.

\section{In vitro fertilisation and embryo culture}

Two bulls were previously tested to determine the optimal sperm concentration achieving maximum fertilisation with minimal polyspermy. (a) A first bull was then used for IVF and embryo transfer experiments, while (b) the second was used for the production of blastocysts that were used for sex determination.

(a) For the experimental groups, the semen samples were sorted according to the protocol described in the experiment 5 (Table 1). For the control groups, after thawing and Percoll gradients separation, half volume of the semen samples prepared for sorting were pooled and kept at room temperature as long as the time required for sorting. Thus, oocytes of control groups and sorted groups were fertilised at the same time, approximately 3-4 $\mathrm{h}$ after thawing.

After sorting, semen samples were brought to a final volume of $2 \mathrm{ml}$ with bicarbonate-buffered TALP IVF heparin-free medium and centrifuged for $10 \mathrm{~min}$ at $500 \mathrm{~g}$. The supernatants were removed and the sperm pellets resuspended with $1 \mathrm{ml}$ of the same IVF medium in which $1 \mu \mathrm{g} / \mathrm{ml}$ heparin was added. After being centrifuged for $10 \mathrm{~min}$ at $500 \mathrm{~g}$, the pellets were counted and the volume adjusted to give a concentration of approximately $0.4-0.6 \times 10^{6} \mathrm{sperms} / \mathrm{ml}$. An aliquot of $10 \mu \mathrm{l}$ sperm solution was immediately pooled for real-time PCR analysis. After the addition of a solution of penicillamine, hypotaurine and epinephrine (PHE, 100X; Rosenkrans et al. 1993), the sperm suspension was poured into wells in $50 \mu \mathrm{l}$ microdrops and covered with mineral oil. Thereafter, 20 matured oocytes per drop were added and incubated in $5 \% \mathrm{CO}_{2}$ and $5 \% \mathrm{O}_{2}$ in humidified air at $38.5{ }^{\circ} \mathrm{C}$ (Galli et al. 2001). After $18-20 \mathrm{~h}$, the presumptive fertilised eggs were vortexed in HEPES-buffered h-SOF medium to remove the cumulus cells. Denuded zygotes were cultured in $300 \mu \mathrm{l}$ bicarbonate-buffered SOF medium, and on day 4 , half of the medium was renewed. The day 6 of culture, half volume was replaced with TCM 199 (Ponderato et al. 2001) supplemented with BSA (no. A8806, fatty acid free, Lot 81 K7600, Sigma). Blastocysts were evaluated at days 7 and 8 and were either cryopreserved or immediately transferred into synchronised recipients. Polyspermia was assessed by fixing part of the non-cleaved eggs on a clean glass slide in 3:1 ethanol:acetic acid overnight. The fixed oocytes were then stained with lacmoid and analysed under phase contrast microscopy.

The fertilisations were repeated in 12 trials in which semen samples sorted for the X-chromosome were used.

(b) A series of blastocysts were obtained using the protocol of IVF/IVC as described earlier in four replicates using X-sorted semen samples and non-sorted control samples, and the sex was then determined.

\section{Sex determination of in vitro produced blastocysts}

For sex determination, blastocysts derived from oocytes fertilised with X-sorted sperms and non-sorted control samples were subjected to PCR analysis. The analysis was conducted according to the method described by Peura et al. (1991) using one set of bovine Y-chromosome-specific primers, one set of

Table 1 Effects of different staining procedures on sorting purity of frozen-thawed bovine sperm samples assessed by real-time PCR analysis.

\begin{tabular}{lccccc}
\hline Experiment & Replicates no. & Conc. $\mathbf{H 3 3 3 4 2}(\mu \mathrm{g} / \mathrm{ml})$ & $\begin{array}{c}\text { Temperature of } \\
\text { incubation }\end{array}$ & \% X mean \pm s.D. & \% Y mean \pm s.D. \\
\hline 1 & 7 & 100 & Room & Not detectable & Not detectable \\
2 & 6 & 100 & $37^{\circ} \mathrm{C}$ & $67 \pm 16$ & $60 \pm 10$ \\
3 & 6 & 500 & Room & $73 \pm 6$ & $73 \pm 6$ \\
4 & 13 & 500 & $37^{\circ} \mathrm{C}$ & $91 \pm 2$ & $74 \pm 10$ \\
$5^{\mathrm{a}}$ & 14 & 500 & $37^{\circ} \mathrm{C}$ & $50 \pm 2$ \\
Control $^{a}$ & 46 & - & - & - \\
\hline
\end{tabular}

In experiment 5 , the Percoll gradients procedure was modified by the presence of $0.6 \%$ BSA in the $90 \%$ fraction.

${ }^{a}$ These experiments were conducted using frozen-thawed semen of two different bulls. 
bovine DNA-specific primers and agarose gel electrophoresis.

\section{Semen analysis by real-time PCR}

The percentages of $\mathrm{X}$ - and $\mathrm{Y}$-chromosomes in sorted semen samples were estimated by a protocol developed and validated in our laboratory (Bongioni et al. 2002). The procedure is briefly described as following.

Bovine specific X- and Y-chromosome primers and probes were designed on PLP and SRY genes respectively, and the probes were labelled with two different fluorochromes.

A certified standard curve was obtained using a mixture of two plasmids containing the $X-$ and Y-amplicons. To this aim, the plasmids with an initial concentration of about $10^{6}$ molecules and a precise ratio of $1: 1$, were used to prepare five mixtures of scalar dilutions with factor 10 (from 100 to $0.01 \%$ ) and subjected to PCR amplification. By plotting the $C_{\mathrm{t}}$ values (first cycle at which the products of amplification were first detectable) obtained by the two DNA fragments of SRY and PLP genes, against the logarithm of each dilution point of the mix of plasmids, two regression plots were generated (Fig. 1).

For semen analysis, the DNA extracted from the samples was amplified in real-time PCR and two distinct ampliplots were generated: the first plot derived from the amplification of the $\mathrm{X}$-chromosome and the second from the amplification of the Y-chromosome (Fig. 2). The two ampliplots were used to determine the $C_{\mathrm{t}}$ values that were consequently used to derive the percentages of dilution $\left(C_{0} \mathrm{X}\right.$ and $\left.C_{0} \mathrm{Y}\right)$ using the relative standard curve. The percentages of $X$ - and $Y$-bearing sperms in the samples were finally calculated from the ratio between the quantities expressed as percentages of dilutions using the following equation

$\% \mathrm{X}=n /(n+1) \times 100$,

where $n=C_{0 \_} \mathrm{X} / C_{0} \_\mathrm{Y}$.

As the sex ratio of sperm samples was derived from two relative concentrations, an absolute quantification of DNA in the samples was not required. Rather, for a correct sex ratio determination, it was fundamental that, after amplification, the DNA provided $C_{\mathrm{t}}$ values that were within the range of the reference standard curves.

\section{Statistical analysis}

Differences between the experimental groups were valued by the Chi-square test.

\section{Results}

\section{Evaluation of different sperm presorting stain treatments}

The effect of different staining procedures on the ability to sort the semen samples into X- and Y-chromosome bearing sperms, as assessed by real-time PCR, is presented in Table 1.

In experiment 1, it was not possible to identify the population corresponding to X- and Y-bearing spermatozoa and consequently to separate them, in none of the trials. Increasing the temperature of incubation to $37^{\circ} \mathrm{C}$ (experiment 2) had beneficial effect for the identification of the fluorescence signal. Nevertheless, this procedure was not applicable due to the great variability among the days (in three occasions the presumptive sorted samples after real-time PCR analysis showed values of enrichment similar to the non-sorted control samples) and the

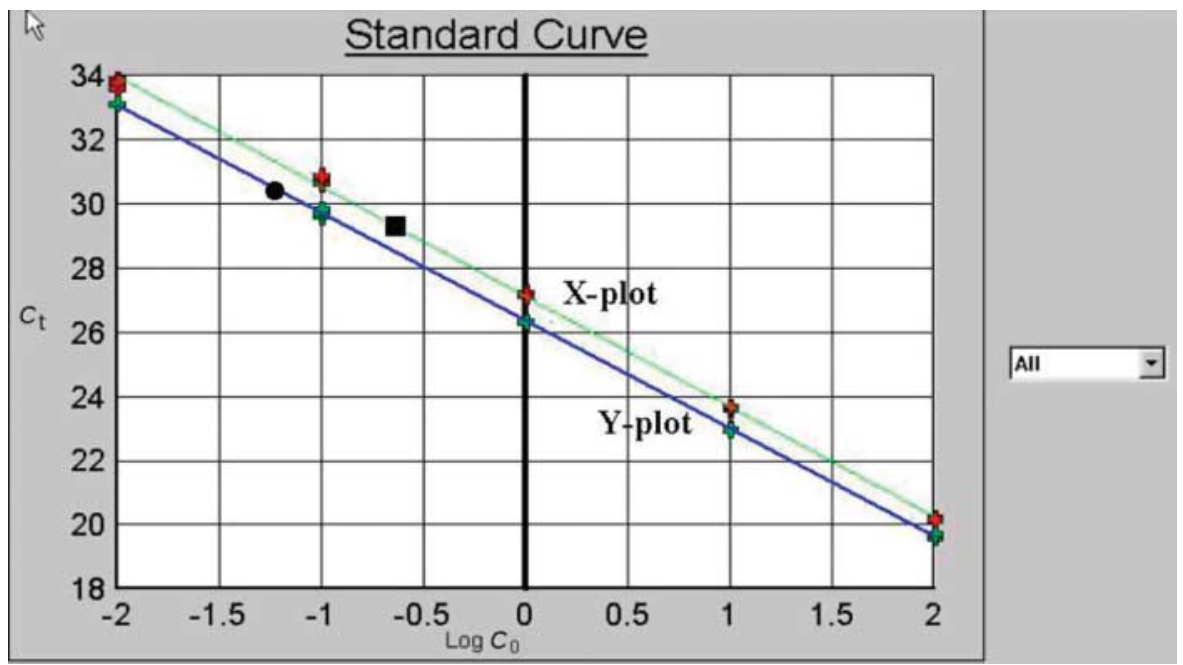

Figure 1 Standard reference curves $(X-$ and Y-plots) generated by plotting $C_{\mathrm{t}}$ values of the SRY and PLP gene fragments inserted in the plasmids against the logarithm of each dilution point percentage $\left(\log C_{0}\right)$. The interpolation of the $C_{\mathrm{t}-\mathrm{X}}(\boldsymbol{\square})$ and $C_{\mathrm{t}} \mathrm{Y}(\boldsymbol{\bullet}$ values obtained by semen sample analysis on the two respective regression plots allowed to obtain the relative amount of $X$ and Y-chromosome content. 


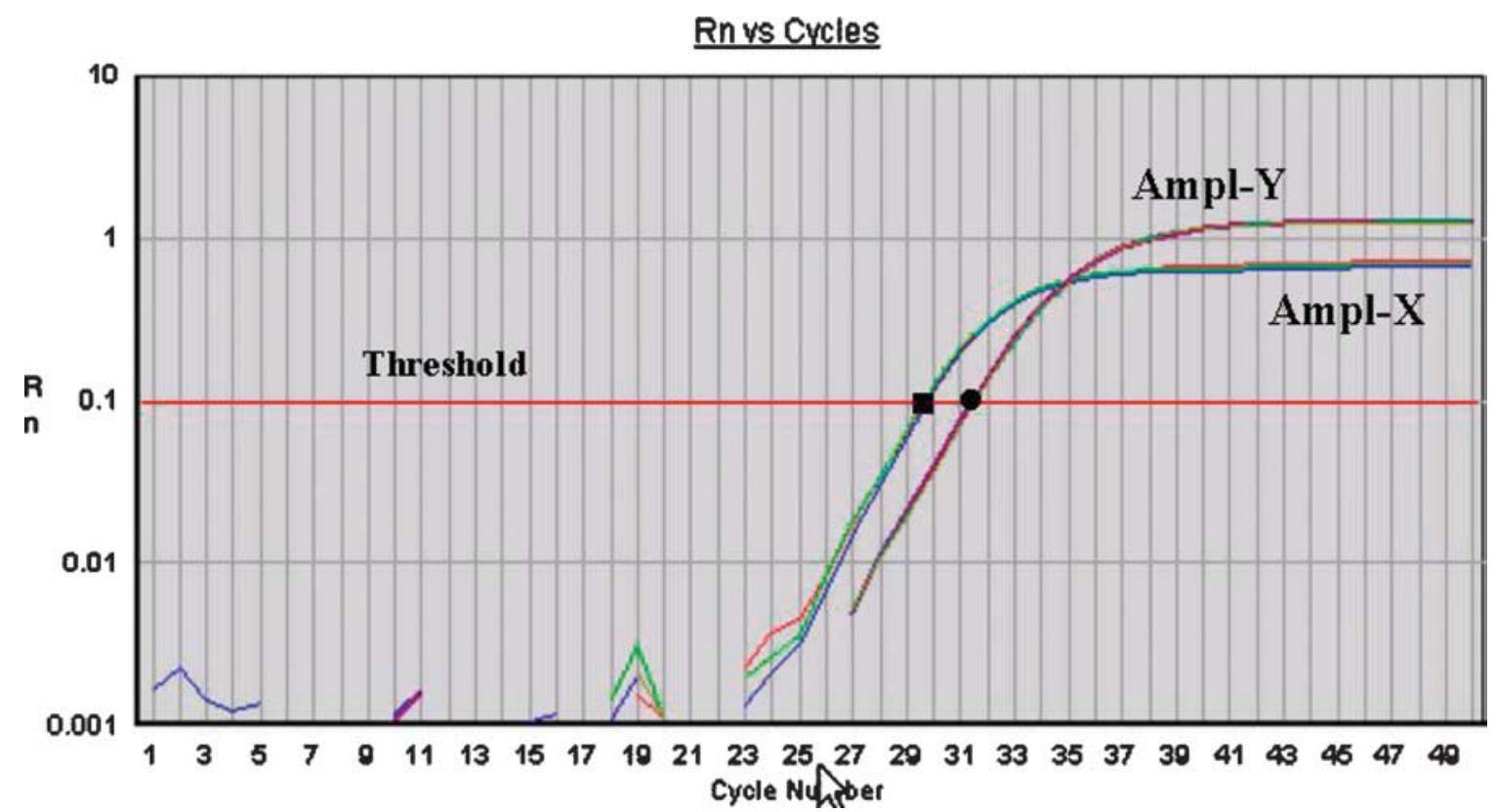

Figure 2 Example of fluorescence amplification plots deriving from real-time PCR analysis of a semen sample sorted for the X-chromosome. The intercept between the threshold and the fluorescence plots allowed to compute the $C_{\mathrm{t}-\mathrm{X}}$

very little efficiency of sorting. A main problem evidenced during these trials was a shifting of the fluorescence signal, which prevented the maintenance of the parameters set for the sorting procedure. Therefore, increasing concentrations of dye were tested. To this aim, a series of samples were only cytometrically analysed without physical separation, and the fluorescence histograms were monitored during a period of $2 \mathrm{~h}$. In these trials, the concentration of $\mathrm{H} 33342$ was tested at 200, 300, 400 and $500 \mu \mathrm{g} / \mathrm{ml}$, and the highest concentration, revealed to be optimal for providing a good level of saturation ensuring a stable and durable signal. This concentration was then tested for sorting efficiency at two temperatures (experiments 3 and 4) and the accuracy was evaluated by real-time PCR. In experiment 3, a significant improvement in the sorting efficiency and a reduction of the day-to-day variability were obtained, but the percentage of X-enrichment never exceeded $78 \%$. The problem related to the shifting of signal did not occur during all the trials of experiment 4, in which the concentration of $500 \mu \mathrm{g} / \mathrm{ml} \mathrm{H} 33342$ and the incubation for $15 \mathrm{~min}$ at $37^{\circ} \mathrm{C}$ provided a clear resolution of the two peaks corresponding to $X$ - and Y-populations and a stable maintenance of the signal during the sorting procedure. These conditions increased the purity of X-bearing sperms, although a considerable variability between days was retained ranging from 77 to $96 \%$. The presence of sperm agglutination after Percoll gradients separation, reported discontinuously during the sorting sessions, was considered to be the major source of the observed variability. In the subsequent experiment 5, in which two different bulls were used, with the exception of one sorting event $(85 \%)$, all the trials led to enrichments in X-chromosome ranging from 90 to $95 \%$ and an acceptable low variability, demonstrating constant high purity and repeatability. In particular, for the first bull the value of X-chromosome enrichment, expressed as mean \pm S.D., was $92 \pm 1$ and for the second bull, it was $91 \pm 3$. Nevertheless, less precision of sorting and greater variability still persisted in the samples sorted for the Y-chromosome, where values of $80 \pm 7$ and $76 \pm 6$ were reported for the first and second bull respectively. Statistical analysis showed no difference in semen separation success between the two bulls, during the 14 repetitions of this trial. For experiment 5 , values of $X$ and $Y$ enrichments are presented in Table 1 as results obtained with both the bulls.

\section{In vitro fertilisation and embryo culture}

Fertilisation rates and embryo development are presented in Table 2. Although the fertilisation rate in control groups was higher than in sorted groups (48 vs $40 \%, P<0.01)$, blastocysts formation in the control and the sorted groups was not statistically different (24 and $27 \%$ of the cleaved respectively).

No polyspermic fertilisations were reported in the groups of oocytes fertilised with sorted samples in which the sperm concentration was approximately $0.4-0.6 \times$ $10^{6} \mathrm{sperms} / \mathrm{ml}$. In the non-sorted control groups, that in these trials were routinely inseminated with the optimised concentration of $0.2 \times 10^{6} \mathrm{sperms} / \mathrm{ml}$, increasing the concentration to $0.4 \times 10^{6}$ sperms $/ \mathrm{ml}$ (similar to those used for the sorted samples) during the preliminary tests, led to $54 \%$ of polyspermia. 
Table 2 Fertilisation rates and embryo development of bovine oocytes fertilised with frozen-thawed cytometrically sorted spermatozoa and nonsorted control samples.

\begin{tabular}{|c|c|c|c|c|c|c|c|}
\hline \multirow[b]{3}{*}{ Replicates $(n)$} & \multirow[b]{3}{*}{$\% \mathrm{X}$ mean \pm s.D. } & \multirow[b]{3}{*}{ Oocytes number } & \multirow{3}{*}{$\begin{array}{c}\text { Oocytes } \\
\text { undergoing } \\
\text { cleavage no. }(\%)\end{array}$} & \multicolumn{2}{|c|}{ Day 7} & \multicolumn{2}{|c|}{ Day 8} \\
\hline & & & & \multicolumn{2}{|c|}{$\begin{array}{l}\text { Blastocysts/ } \\
\text { cleaved no. (\%) }\end{array}$} & \multirow{2}{*}{$\begin{array}{c}\text { Blastocysts/ } \\
\text { cleaved no. (\%) }\end{array}$} & \multirow{2}{*}{$\begin{array}{l}\text { Blastocysts/ } \\
\text { oocytes (\%) }\end{array}$} \\
\hline & & & & Grade I & Total & & \\
\hline X-sorted (12) & $90 \pm 2$ & 440 & $175(39.7)^{\mathrm{a}}$ & $24(13.7)$ & $33(18.8)$ & $48(27.4)$ & (10.9) \\
\hline Control (6) & $50 \pm 2$ & 233 & $112(48)^{\mathrm{b}}$ & 13 (11.6) & 21 (18.7) & $27(24.1)$ & (11.6) \\
\hline
\end{tabular}

Values with different letters within columns differ significantly, $P<0.01$ (Chi-square). Sorted samples were used at concentrations of $0.4-0.6 \times 10^{6} \mathrm{sperm} / \mathrm{ml}$. Control oocytes were inseminated at $0.2 \times 10^{6} \mathrm{sperm} / \mathrm{ml}$ and after Percoll, the non-sorted samples were stored at room temperature as long as the time required for sorting.

The volume of $10 \mu \mathrm{l}$ that was sampled from the IVF sperm suspensions and used for real-time PCR analysis was adequate for the determination of sorting purity in all the IVF experiments. Enrichments in X-chromosome-bearing sperms of approximately $90 \%$ were repeatedly obtained.

Transfer of 12 fresh sexed embryos into six synchronised recipients (two embryos per recipient) resulted in four pregnancies. Embryo loss within day 60 occurred in two of the pregnant animals, while the two remaining animals carried the pregnancy to term giving birth to two female calves.

\section{Sex confirmation of blastocysts}

The results of PCR analysis of in vitro produced embryos are shown in Table 3. The analysis of 30 blastocysts derived from oocytes fertilised with semen samples sorted for the X-chromosome, confirmed 26 blastocysts to be correctly sexed $(87 \%)$.

\section{Discussion}

According to the protocol described firstly by Johnson \& Pinkel (1986) and worldwide used for fresh semen sexing, sperm staining procedure is performed using low concentration of $\mathrm{H} 33342$ for extended period of times, with temperatures ranging from 34 to $37^{\circ} \mathrm{C}$. Unfortunately, similar conditions have never allowed a clear resolution of the sexual chromosomes when frozenthawed semen was used. To address this issue, the modifications of sperm treatments were introduced in the attempt to overcome the negative response to the staining procedures observed with frozen-thawed semen. A better resolution of the sexual chromosomes was achieved by quenching the fluorescence of the dead sperms with Percoll in thawed samples after the removal of cryoprotectants by simple washing procedures, even though the sorting efficiency did not exceed $80 \%$ (Stap et al. 1998). In the same work, the authors reported no beneficial effects in the use of Percoll for physically separating the dead from the living sperms by centrifugation through gradients. In other studies conducted on bull and ram frozen-thawed semen, Percoll gradients separation together with the use of food dye as a quencher for dead sperms led to high purity of sorting, although requiring concentration of $\mathrm{H} 33342$ higher than those necessary for fresh semen. Nevertheless, a decline in the motility of these spermatozoa has been observed during the prolonged incubations at $34{ }^{\circ} \mathrm{C}$ required for DNA staining. On the basis of this consideration, we focused our study on the possibility to reduce the time required for staining although providing a good resolution of the fluorescence signal. In the same time, we also investigated the possibility for incubating the sperms at room temperature avoiding continuous changes of environmental conditions. The data obtained demonstrate that the high sorting purity of frozen-thawed samples can be achieved even after a marked reduction in the incubation time, using Percoll separation and increased concentration of H33342. Nevertheless, the dynamic of sperm dye incorporation revealed to be not adequate when the incubation was carried out at room temperature.

Adding $0.6 \%$ BSA in the $90 \%$ Percoll fraction significantly increased the purity of sorted samples, thanks to the beneficial effect in preventing the agglutination of spermatozoa that was reported occasionally and associated to different bulls or semen batches. The video recording of the sperm samples just prior (after Percoll separation) and after the staining procedures, clearly demonstrated the efficacy of this

Table 3 Sex determination by PCR analysis of bovine blastocysts derived from four different trials using $X$-sorted semen samples, and control blastocysts obtained by non-sorted samples.

\begin{tabular}{lcccc}
\hline $\begin{array}{l}\text { X-sorted } \\
\text { semen } \\
\text { enrichment }\end{array}$ & Oocytes no. & $\begin{array}{c}\text { Blastocysts } \\
\text { analysed no. }\end{array}$ & $\begin{array}{c}\text { Males ơ; } \\
\text { no. }\end{array}$ & $\begin{array}{c}\text { Females } \% ; \\
\text { no. }\end{array}$ \\
\hline $87 \%$ & 80 & 8 & 2 & 6 \\
$92 \%$ & 80 & 7 & 0 & 7 \\
$93 \%$ & 80 & 6 & 0 & 6 \\
$88 \%$ & 80 & 9 & 2 & 7 \\
$\begin{array}{l}\text { Total blastocysts } \\
\text { Sexed }\end{array}$ & & 30 & $4(13 \%)$ & $26(87 \%)$ \\
Control & & 29 & $12(41 \%)$ & $17(58 \%)$ \\
\hline
\end{tabular}


modification over the standard Percoll gradients and washing procedures. Regarding the problem of sperm clustering and its negative effect on sorting, the removal of agglutinated sperms by filtering the samples through large pore size filters was suggested by other authors, although this practice results in a loss of sperm number, which could be otherwise available for sorting.

In experiment 5, where the optimised protocol was used, less purity and more variability was retained, while sorting for the Y-chromosome. One possible explanation, could be the particular response of frozenthawed semen to staining procedures, that still represents one of the main problems. In particular, a non-uniform staining to dyes of frozen semen has been reported (Weigel 2004). This could result in the minor purity of Y-chromosome bearing sperms as a consequence of the minor DNA content and fluorescence emission. On the other hand, this problem was not reported with $\mathrm{X}$-chromosome sperms that, maybe because of a more uniform response to staining, could be sorted with purities similar to those obtained with fresh semen.

During these trials, the real-time PCR for the analysis of sexed samples obtained after different treatments revealed to be an optimal instrument for supporting these studies, providing a rapid and reliable verification of the effects of various protocol modifications. Furthermore, when sorted semen was used for IVF, this technique demonstrated a very high level of sensitivity and accuracy. In fact, a minimum volume of sperm samples, $10 \mu \mathrm{l}$ containing 4000-5000 spermatozoa, provided yields of DNA largely included in the orders of linear dynamic range of the references. This great advantage permitted to estimate the sorting purity by directly sampling the sperm suspensions used for IVF involving only a negligible loss of viable and potentially useful spermatozoa, in a situation in which the resorting analysis could not be used because of the larger number of sperm required for this application.

In a previous work of Hollinshead et al. (2004a), in which ram frozen semen was sorted and used in IVF, the blastocyst formation of oocytes fertilised with sorted sperms appeared to be favoured with respect to the oocytes inseminated with non-sorted control sperms. In different studies, aimed at characterising sperm factors related to the fertility (Eid et al. 1994, Comizzoli et al. 2000), it was postulated that embryo development can be drastically affected by paternal factor influencing the G2-phase duration and the onset of the M-phase and that the progress to blastocyst stage would be more advantaged for oocytes fertilised by high in vivo fertility bulls. Similarly, it can be hypothesised that artificial manipulations, such as the sorting of frozen-thawed samples, could result in a selection towards the more viable sperm population. Differently from our work, in which the embryo development was not affected by sperm sorting procedure, the superior embryo development reported by Hollinshead et al. (2004a) after sperm sorting could probably be due to the second cycle of freezing, which determined a further selection of the semen, conducted after the frozen semen was thawed and sorted. Furthermore, a similar effect was not evidenced, despite in our work, the sorted sperms were used at very low concentration (nearly $0.4 \times$ $10^{6} \mathrm{sperms} / \mathrm{ml}$ ) with respect to $1 \times 10^{6} \mathrm{sperms} / \mathrm{ml}$ used in the above-cited work, considering that the minor sperms:oocyte ratio was expected to favour the more viable sperms and enhance the difference of IVF between low and high fertility bulls (Puglisi et al. 2004).

A previous study aimed at the evaluation of mutagenic effects of Hoechst 33342 conducted on human spermatozoa (Watkins et al. 1996) revealed possible risks for mutagenesis at high concentrations of dye $(>900 \mu \mathrm{M})$ after $24 \mathrm{~h}$ of exposition. In our study, the high concentrations of dye were used for no more than $15 \mathrm{~min}$, after which sperms were diluted to a concentration that, in the same work, has been demonstrated to be non-mutagenic even after 24-h incubation. Higher concentration $(2240 \mu \mathrm{M})$ and 1 -h incubation have also been used for sorting experiments (Lu et al. 1999) and blastocyst formation was not affected by staining procedure. Nevertheless, more detailed studies based on molecular genetic approaches would be beneficial to address this kind of investigations, in order to exclude completely a potentially detrimental effect of the treatment.

This is the first report, in our knowledge, of offspring derived by transferring in vitro produced bovine embryos obtained by sorting frozen-thawed sperms. In this work, the high concentration of $\mathrm{H} 33342$ required for an optimal resolution of the sexual chromosomes, represented the major concern for a practical use of this procedure. However, if the incubation time is minimised to $15 \mathrm{~min}$, this concentration seems to be compatible with normal embryo survival and fetal development. However, due to the limited number of embryo transfers conducted in this study and the embryo losses that we have evidenced, further studies to be conducted on embryos or offspring are required in order to exclude any possible mutagenic effect of high concentrations of $\mathrm{H} 33342$.

\section{Acknowledgements}

This work was supported by the RAIZ research project of M.I.P.A.F. The authors declare that there is no conflict of interest that would prejudice the impartiality of this scientific work.

\section{References}

Bodmer M, Jannett F, Hassig M, Daas N, Reichert P \& Thun R 2005 Fertility in heifers and cows after low dose insemination with sexsorted and non-sorted sperm under field conditions. Theriogenology 64 1647-1655. 
Bongioni G, Parati K \& Galli A 2002 New real time PCR method for quantification of sex ratio in bull semen. (15th European Colloquium on Animal Cytogenetics and Gene Mapping; Sorrento, Italy 2-4 June 2002) Chromosome Research 1034.

Checa ML, Dunner S \& Cañón J 2002 Prediction of $X$ and $Y$ chromosome content in bovine sperm by using DNA pools through capillary electrophoresis. Theriogenology 58 1579-1586.

Comizzoli P, Marquant-Le Guienne B, Heyman Y \& Renard JP 2000 Onset of the first S-phase is determined by paternal effect during the G1-phase in bovine zygotes. Biology of Reproduction 62 1677-1684.

Eid LN, Lorton SP \& Parrish JJ 1994 Paternal influence on S-phase in the first cell cycle of the bovine embryo. Biology of Reproduction $\mathbf{5 1}$ 1232-1237.

Galli C, Crotti G, Notari C, Turini P, Duchi R \& Lazzari G 2001 Embryo production by ovum pick up from live donors. Theriogenology $\mathbf{5 5}$ 1341-1357.

Grossfeld R, Klinc P, Sieg B \& Rath D 2005 Production of piglets with sexed semen employing a non-surgical insemination technique. Theriogenology 63 2269-2277.

Habermann FA, Winter A, Olsaker I, Reichert P \& Fries R 2005 Validation of sperm sexing in the cattle (Bos taurus) by dual colour fluorescence in situ hybridization. Journal of Animal Breeding and Genetics 122 22-27.

Hollinshead FK, Evans G, Evans KM, Catt SL, Maxwell WMC \& O'Brien JK 2004a Birth of lambs of a pre-determined sex after in vitro production of embryos using frozen-thawed sex-sorted and re-frozen-thawed ram spermatozoa. Reproduction 127 557-568.

Hollinshead FK, O'Brien JK, Maxwell WMC \& Evans G 2004b Assessment of in vitro sperm characteristics after flow cytometric sorting of frozen-thawed bull spermatozoa. Theriogenology 62 958-968.

Johnson LA 2000 Sexing mammalian sperm for production of offspring: the state-of-the-art. Animal Reproduction Science 60-61 93-97.

Johnson LA \& Pinkel D 1986 Modification of a laser-based flow cytometer for high resolution DNA analysis of mammalian spermatozoa. Cytometry 7 268-273.

Lindsey AC, Schenk JL, Graham JK, Bruemmer JE \& Squires EL 2002 Hysteroscopic insemination of low numbers of flow sorted fresh and frozen thawed stallion spermatozoa. Equine Veterinary Journal 34 $121-127$
Lu KH, Cran DG \& Seidel GE Jr 1999 In vitro fertilization with flowcytometrically-sorted bovine sperm. Theriogenology 52 1393-1405.

Peura T, Hyttinen LM, Turunen M \& Jänne J 1991 A reliable sex determination assay for bovine preimplantation embryos using the polymerase chain reaction. Theriogenology 35 547-555.

Ponderato N, Lagutina I, Crotti G, Turini P, Galli C \& Lazzari G 2001 Bovine oocytes treated prior to in vitro maturation with a combination of butyrolactone I and roscovitine at low doses maintain a normal developmental capacity. Molecular Reproduction and Development 60 579-585.

Puglisi R, Balduzzi D \& Galli A 2004 In vitro sperm penetration speed and its relationship with in vivo bull fertility. Reproduction in Domestic Animals 39 424-428.

Rosenkrans CF Jr, Zeng GQ, McNamara GT, Schoff PK \& First NL 1993 Development of bovine embryos in vitro as affected by energy substrates. Biology of Reproduction 49459.

Seidel GE Jr, Schenk JL, Herickhoff LA, Doyle SP, Brink Z, Green RD \& Cran DG 1999 Insemination of heifers with sexed sperm. Theriogenology 52 1407-1420.

Stap J, Hoebe RA, Merton JS, Haring RM, Bakker PJM \& Aten JA 1998 Improving the resolution of cryopreserved $\mathrm{X}$ - and Y-sperm during DNA flow cytometric analysis with the addition of percoll to quench the fluorescence of dead sperm. Journal of Animal Science $\mathbf{7 6}$ 1896-1902.

Watkins AM, Chan PJ, Kalugdan TH, Patton WC, Jacobson JD \& King A 1996 Analysis of the flow cytometer stain Hoechst 33342 on human spermatozoa. Molecular Human Reproduction 2 709-712.

Weigel KA 2004 Exploring the role of sexed semen in dairy production systems. Journal of Dairy Science 87 120-130.

Welch GR \& Johnson LA 1999 Sex preselection: laboratory validation of the sperm sex ratio of flow sorted X- and Y-sperm by sort reanalysis for DNA. Theriogenology 52 1343-1352.

Received 8 March 2006

First decision 7 April 2006

Revised manuscript received 25 May 2006

Accepted 20 June 2006 